\title{
UNCERTAINTY PRINCIPLES IN TERM OF SUPPORTS IN HANKEL WAVELET SETTING
}

\author{
S. HKIMI AND S. OMRI*
}

Abstract. Uncertainty principles in term of supports, namely Amrein-Berthier and LogvinenkoSereda theorems are proved for the continuous Hankel wavelet transform.

Mathematics subject classification (2020): 42A38, 35S30, 42B10.

Keywords and phrases: Hankel wavelet transform, uncertainty principle, annihilating set, AmreinBerthier theorem, Logvinenko-Sereda theorem.

\section{REFERENCES}

[1] W. O. AMREIN AND A. M. BERTHIER, On support properties of Lp-functions and their Fourier transforms, J. Funct. Anal. Vol 24 (1977): 258-267.

[2] G. Andrews, R. Askey and R. Roy, Special functions, New York (NY): Cambridge Univ Press; 1999.

[3] C. BACCAR, Uncertainty principles for the continuous Hankel Wavelet transform, Integral Transforms Spec. Funct. Vol 27 (2016), num 6: 413-429.

[4] I. DAUBECHIES, Ten lectures on wavelets, Vol. 61. Siam, 1992.

[5] G. B. Folland And A. SitARAm, The uncertainty principle: a mathematical survey, J. Fourier Anal. Appl. Vol 3 (1997): 207-238.

[6] D. GABOR, Theory of communication. Part 1: The analysis of information, Journal of the Institution of Electrical Engineers Vol 93 (1946): 429-441.

[7] S. GHobBer And P. JAming, Strong annihilating pairs for the Fourier-Bessel transform, J. Math. Anal. Appl. Vol 377 (2011), num 2: 501-515.

[8] S. GhobBer And P. Jaming, The Logvinenko-Sereda theorem for the Fourier-Bessel transform, Integral Transforms Spec. Funct. Vol 24 (2013), num 6: 470-484.

[9] S. GHOBBER And P. JAMing, Uncertainty principles for integral operators, Studia Math. Vol 220 (2014), num 3: 197-220.

[10] A. Grossmann And J. Morlet, Decomposition of Hardy functions into square integrable wavelets of constant shape, SIAM J. Math. Anal. Vol 15 (1984), num 4: 723-736.

[11] N. B. HAMADI AND S. OMRI, Uncertainty principles for the continuous wavelet transform in the Hankel setting, Appl. Anal. Vol 97 (2018), num 4: 513-527.

[12] A. J. S. Hamilton, Uncorrelated modes of the non-linear power spectrum, Mon. Not. R. Astron. Soc. Vol 312 (2000), num 2: 257-284.

[13] H. HANKEL, Die Fourier'schen Reihen und Integrale für Cylinderfunctionen, Math. Ann. 8 (1875).

[14] V. HAVIN AND B. JÖRICKE, The uncertainty principle in harmonic analysis, Berlin (BL): Springer Verlag, 1994.

[15] W. HEISENBERG, Über den anschaulichen Inhalt der quantentheoretischen Kinematic und Mechanik, Zeit. Physik, 43, 172 (1927); The Physical Principles of the Quantum Theory (Dover, New York, 1949; The Univ. Chicago Press, 1930).

[16] N. N. LeBedeV, Special functions and their applications, New York (NY): Dover Publications, 1972.

[17] V. N. LOGVINENKO AND Y. F. SEREDA, Equivalent norms in spaces of entire functions of exponential type, Teor. Funktsii. Funktsional. Anal. Prilozhen. Vol 19 (1973): 234-246.

[18] K. Maнато, The composition of fractional Hankel wavelet transform on some function spaces, App. Math. and Computation Vol 337 (2018): 76-86. 
[19] J. Mathews And R. L. WalKer, Mathematical methods of physics, Vol 501, New York (NY): WA Benjamin, 1970.

[20] R. S. Pathak And M. M. Dixit, Continuous and discrete Bessel wavelet transforms, J. Comput. Appl. Math. Vol 160 (2003), num 1: 241-250.

[21] A. Prasad A AND K. MAhato, The fractional Hankel wavelet transformation, Asian-European Journal of Mathematics Vol 8 (2015), num 2: 1550030.

[22] B. RICAUD AND B. TORRSANI, A survey of uncertainty principles and some signal processing applications, Adv. Comput. Math. 40 (2014), no. 3, 629-650.

[23] H. Selim, M. Delgado Prieto, J. Trull, L. Romeral and C. Cojocaru, Laser ultrasound inspection based on wavelet transform and data clustering for defect estimation in metallic samples, Sensors Vol 19 (2019), num 3: 573-585.

[24] M. STEPhane, A wavelet tour of signal processing, The Sparse Way, 1999.

[25] U. B. ÜnAlmis, Fractional Hankel and Bessel wavelet transforms of almost periodic signals, J. Inequal. Appl. (2015): 388.

[26] E. WilczoK, New Uncertainty Principles for the Continuous Gabor Transform and the Continuous Wavelet Transform, Doc. Math. Vol 5 (2000): 201-226.

[27] W. A. WoycZyns Ki, Uncertainty Principle and Wavelet Transforms, in: A First Course in Statistics for Signal Analysis. Statistics for Industry, Technology, and Engineering, Birkhäuser, Cham. 